\title{
Blurred Image Restoration at Low Resolution
}

\author{
Lidong Huang, Xuewen Tan*, Xiaozhou Chen and Yanfang Zhao \\ School of Mathematics and Computer Science, Yunnan Minzu University, Kunming 650500, P. R. China \\ ${ }^{*}$ Corresponding author
}

\begin{abstract}
This paper through the use of super- resolution image reconstruction technology, it is using multiple low resolution images of the same scene, the relative movement between the information they are integrated into a single frame in the high resolution image, and the initial high-resolution images of the recovery and denoising can get a relatively clear high resolution image, and the frame contains a single frame can't reflect the details of the low resolution pictures.
\end{abstract}

Keywords—image degradation; image registration; image interpolation; image restoration; image denoising

\section{INTRODUCTION}

Image super resolution reconstruction is a kind of image processing technology that uses a computer to process a low resolution image or image sequence and restores a high resolution image. HR means that the image has a high pixel density, which can provide more details, these details often play a key role in the application [1][2][3][4][5][6]; The method of super-resolution reconstruction is Regularized reconstruction method: The process of image reconstruction; The process of image Reconstruction; Interpolation method for non-uniform spatial samples; Iterative back projection (IBP) method; Set theory reconstruction method (convex projection POCS); Statistical reconstruction methods (maximum posterior probability MAP and maximum likelihood estimation ML)[9][10][11][12]. Based on the above discussion, Based on super resolution image reconstruction technology, it is the use of low resolution images of the same scene, they are integrated into the relative motion between the high resolution image of single frame information, and to recover the initial noise of high resolution images, we can get a clear high resolution image, and comprises a frame a single framework does not reflect the low resolution image details.

\section{MATHEMATICAL MODEL}

\section{A. Image Acquisition and Digitization}

Selecting a frame of the appropriate image, and make this image gray scale, it can get discretized digital image. If the resolution of a frame of plane static image is $x \cdot y$, let the function $f(x, y)$ denote the frame image, where the function value $f(x, y)$ is the pixel value of the image. From the monochrome image $0 \leq f(x, y) \leq 255$. ( $x, y$ ) is the coordinates of the corresponding pixel, that is, $x$ represents the width of the image, $y$ represents the length of the image. The corresponding digital image can be represented by a two-dimensional matrix.

$$
f(x, y)=\left[\begin{array}{cccc}
f(1,1) & f(1,2) & \ldots \ldots & f(1, y) \\
f(2,1) & f(2,2) & \ldots \ldots & f(2, y) \\
\ldots \ldots & \ldots \ldots . & \ldots \ldots & \ldots \ldots . \\
f(x, 1) & f(x, 2) & \ldots \ldots & f(x, y)
\end{array}\right]
$$

\section{B. Image Degradation}

In the process of image acquisition, the image is usually subject to various factors of interference caused by degradation of image quality. The main causes of image degradation are motion blurred[3], external environmental factors, imaging systems, noise and so on. So, we need to establish the degenerate mathematical model of the image to reflect the mapping relationship between the low-resolution image and the high-resolution target image, and then, make the sequence of low-resolution images for super-resolution reconstruction.

1) Linear Model

$$
T_{k}=D_{k} M_{k} F_{k} S+N_{k} \quad k=1,2,3 \cdots n
$$

This model is widely considered a degenerate model $D_{k}$ represents the sample matrix corresponding to the k-th image. That is the process of obtaining the low-resolution image by the high-resolution image. $M_{k}$ represents the fuzzy matrix corresponding to the $k$-th image. $F_{k}$ represents the motion transformation matrix corresponding to the $k$-th image, which is the geometric deformation between the observed image interpolation and the original high-resolution image. $S$ is high-resolution image, $N_{k}$ is noise vector, $T_{k}$ is the vector of the $k$-th image in low-resolution image. In addition, this equation can be transformed into a matrix in the following form:

$$
\begin{gathered}
{\left[\begin{array}{c}
T_{1} \\
T_{2} \\
\vdots \\
T_{n}
\end{array}\right]=\left[\begin{array}{c}
D_{1} M_{1} F_{1} \\
D_{2} M_{2} F_{2} \\
\vdots \\
D_{n} M_{n} F_{n}
\end{array}\right] S+\left[\begin{array}{c}
N_{1} \\
N_{2} \\
\vdots \\
N_{n}
\end{array}\right]} \\
T=G S+N \quad G=\left(G_{1}, G_{2}, G_{3} \cdots G_{n}\right)^{T} \quad G_{i}=D_{i} M_{i} F_{i}, i=1,2,3 \cdots n
\end{gathered}
$$

2) Using the Convolution Formula to Establish Degenerate Model

The original high frequency image $i(x, y)$ can form degraded image $f(x, y)$ by the effort of degenerate operator or degraded system $h(x, y)$.The degenerate operator or degraded system is the mathematical model to be established. Assuming that the degraded system is invariant in linear and spatial systems, that is, linear and unchanged position. The mode in the airspace is: 


$$
g(x, y)=i(x, y) * h(x, y)+n(x, y)
$$

This model uses convolution formula because the convolution in the time domain corresponds to the product in the frequency domain. The above formula after Fourier Transform in the frequency domain is:

$$
G(x, y)=I(x, y) * H(x, y)+N(x, y)
$$

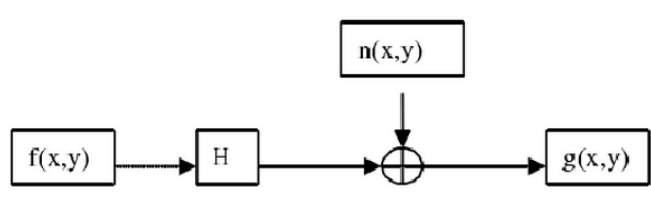

FIGURE I. FTHE IMAGE IN THE PROCESS OF IMAGE DEGRADATION

\section{Image Registration}

Image registration that is the motion estimation of the image, which is the primary task of super-resolution reconstruction. We have chosen a method based on the gray area that is, using their own gray-scale statistical information to measure the similarity of two images directly in the process of registration. This method is an effective registration algorithm doesn't need extract feature point and classification or other pre-processing. But this algorithm is large and sensitive to noise [6].

\section{1) Grayscale Correlation Method}

\section{a) Normalized mutual information method}

In order to avoid the information matching area due to changes in size lead to miscalculation, so we use normalized mutual information method. Reference image is $f_{1}$, registration image is $f_{2}$, and regard pixel value of the image $f$ as discrete random variable. $H\left(f_{1}\right)$ is the entropy of image $f_{1}, H\left(f_{2}\right)$ is the entropy of image $f_{2} . H\left(f_{1}, f_{2}\right)$ is the mutual information of images $f_{1}, f_{2}$. The specific formula is as follows:

The entropy of image $f_{1}$ :

$$
H\left(f_{1}\right)=-\sum_{i=1}^{k} p\left(a_{i}\right) \log p\left(a_{i}\right)
$$

The entropy of image f2:

$$
H\left(f_{2}\right)=-\sum_{i=1}^{l} p\left(b_{i}\right) \log p\left(b_{i}\right)
$$

The joint entropy of images f1, f2:

$$
H\left(f_{1}, f_{2}\right)=-\sum_{i=1}^{k} \sum_{i=1}^{l} p\left(a_{i}, b_{i}\right) \log p\left(a_{i}, b_{j}\right)
$$

Normalized mutual information:

$$
N M I=\frac{H(x)+H(y)}{H(x, y)}
$$

The grayscale range $a_{i}$ of image $f_{1}$ is $0-255$, the distribution law of discrete random variable is $p\left(a_{i}\right)$, so the distribution column of $a_{i}$ is as show table I.

\section{TABLE I. THE DISTRIBUTION COLUMN OF $a_{l}$}

\begin{tabular}{c|ccccc}
$a_{i}$ & $a_{0}$ & $a_{1}$ & $a_{2}$ & $\ldots \ldots$ & $a_{255}$ \\
\hline$P\left(a_{i}\right)$ & $P\left(a_{0}\right)$ & $P\left(a_{1}\right)$ & $P\left(a_{2}\right)$ & $\ldots \ldots$. & $P\left(a_{255}\right)$
\end{tabular}

The grayscale range $b_{i}$ of image $f_{2}$ is $0-255$, the distribution law of discrete random variable is $p\left(b_{i}\right)$, so the distribution column of $b_{i}$ is:

\section{TABLE II. THE DISTRIBUTION COLUMN OF $b_{\imath}$}

\begin{tabular}{c|ccccc}
$b_{i}$ & $b_{0}$ & $b_{1}$ & $b_{2}$ & $\ldots \ldots$. & $b_{255}$ \\
\hline$P\left(b_{i}\right)$ & $P\left(b_{0}\right)$ & $P\left(b_{1}\right)$ & $P\left(b_{2}\right)$ & $\ldots \ldots$ & $P\left(b_{255}\right)$
\end{tabular}

b) Correlation Coefficient Method:

In order to eliminate the interference of the matching measure with the change of local gray intensity. It can use correlation coefficient method, by calculating to get the result $\operatorname{corr}\left(f_{1}, f_{2}\right)$ of two equal-sized images to measure the degree of matching between the two images. If the $\operatorname{corr}\left(f_{1}, f_{2}\right)$ closer to 1 , said the two images more similar, but $|\operatorname{corr}(\mathrm{f} 1, \mathrm{f} 2)| \leq 1$, The formula is as follows:

$$
\begin{aligned}
& \operatorname{corr}\left(f_{1}, f_{2}\right)=\frac{\operatorname{cov}\left(f_{1}, f_{2}\right)}{\delta_{f 1} \delta_{f 2}} \\
& =\frac{\sum_{i} \sum_{j}\left(f_{1}(i, j)-\mu_{f_{1}}\right)\left(f_{2}(i, j)-\mu_{f_{2}}\right)}{\left[\left(\sum_{i} \sum_{j}\left(f_{1}(i, j)-\mu_{f_{1}}\right)^{2}\right)\left(\sum_{i} \sum_{j}\left(f_{2}(i, j)-\mu_{f_{2}}\right)^{2}\right)\right]^{\frac{1}{2}}}
\end{aligned}
$$

f1 represents the reference image, $f_{2}$ represents the unmatched image, $\operatorname{cov}\left(f_{1}, f_{2}\right)$ represents the covariance of images $f_{1}(i, j)$ and $f_{2}(i, j)$, represents the variance of image, $\mu$ represents the mean value of image.

\section{2) Estimation of Registration Parameters}

The least square method is used to solve the best estimation of image registration. $\omega_{1}, \omega_{2} \ldots \ldots \omega_{n}$ are used to represent the correction of position, $x$ represents mean range(that is the estimated value).

$$
\left\{\begin{array}{l}
x=x_{1}+\omega_{1} \\
x=x_{2}+\omega_{2} \\
\cdots \cdots \cdots \cdots \cdots \\
x=x_{n}+\omega_{n}
\end{array}\right.
$$

This equation satisfies a best condition:

$$
\sum \omega^{2}=\left(x-x_{1}\right)^{2}+\left(x-x_{2}\right)^{2}+\ldots \ldots+\left(x-x_{n}\right)^{2}=\min
$$




$$
\text { Let } \frac{\partial\left(\sum \omega^{2}\right)}{\partial x}=0
$$

Find the $\mathrm{x}$ value that satisfies the above formula.

\section{Image reconstruction}

\section{1) Image interpolation}

The image interpolation is to use the image registration parameter to project the pixel information in each frame of the low resolution image sequence into the high resolution grid, and obtain the initial image of high quality and resolution for the next step.

\section{a) Bilinear Interpolation}

Bilinear interpolation is to use the gray value of four adjacent pixel points $(i, j),(i, j+1),(i+1, j),(i+1, j+1)$. And doing linear interpolation in the horizontal direction and vertical direction. Then get the gray value of the final point. ${ }^{[5]}$

Supposing point $\mathrm{E}$ is the interpolated point, around four black pixels are known pixels. The gray value of point $A$ and point $B$ are recorded as $f(A)$ and $f(B)$, and points $A, B, C$ are in the same line. Here is a formula: The linear interpolation of point $\mathrm{A}$ in the horizontal direction is:

$$
f(A)=\mu f(i, j)+(1-\mu) f(i, j+1)
$$

The linear interpolation of point $\mathrm{B}$ in the horizontal direction is:

$$
f(B)=v f(i+1, j)+(1-v) f(i+1, \mathrm{j}+1)
$$

The gray value of the interpolated point $\mathrm{E}$ is:

$$
\begin{aligned}
& f(E)=f(i+\mu, j+v) \\
& =v f(B)+(1-V) f(A) \\
& =(1-\mu)(1-v) f(i, j) \\
& +(1-\mu)_{V} f(i, j+1) \\
& +\mu(1-v) f(i+1, j)+\mu v f(i+1, j+1)
\end{aligned}
$$

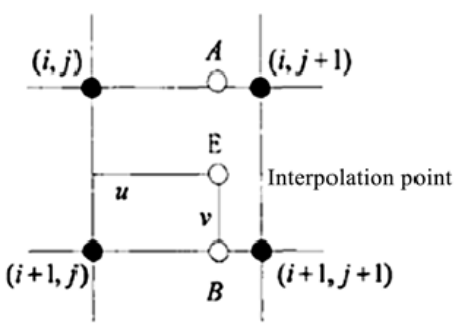

FIGURE II. BILINEAR INTERPOLATION IS TO USE THE GRAY VALUE OF FOUR ADJACENT PIXEL POINTS

\section{b) Nonuniform Interpolation}

According to the results of the registration, put these pixel information into the high-resolution image grid. And make interpolation for these data of uneven interval. Sampling at high resolution grid points to sure the value of each pixel in the high-resolution image, then obtaining an image of high-resolution initial interpolation.

2) Image Restoration ${ }^{[13]}$

a) Inverse Filtering

The degraded image can be expressed as:

$$
g_{i}(x, y)=f_{i}(x, y) * h_{i}(x, y)+n_{i}(x, y)
$$

$g_{i}(x, y)$ represents degraded image. $f_{i}(x, y)$ represents original image. $n_{i}(x, y)$ represents diffusion function. $n_{i}(x, y)$ represents convolution of noise.

After Fourier transform:

$$
\begin{gathered}
G_{i}(x, y)=F_{i}(x, y) H_{i}(x, y)+N_{i}(x, y) \\
F_{i}(x, y)=\frac{G_{i}(x, y)}{H_{i}(x, y)}-\frac{N_{i}(x, y)}{H_{i}(x, y)}
\end{gathered}
$$

$G_{i}(x, y), F_{i}(x, y), N_{i}(x, y)$ are the results of $g_{i}(x, y), f_{i}(x, y)$, $n_{i}(x, y)$,after Fourier transform. If you know the degradation of image, the diffusion function, the noise of the Fourier transform, you can find the Fourier transform of the original image. At the last, we can obtain the corresponding original image by Fourier inversion $g_{i}(x, y)$.

\section{b) Least Squares Filter}

Find an estimated value $\hat{f}$, make the function $\|\mathrm{Q} \hat{f}\|^{2}$ $=\|\mathrm{n}\|{ }^{2}$ minimize, this is a degraded image restoration method, this method is commonly using Lagrangian multiplier algorithm to solve problem. That is to find a minimum of the following functions. ${ }^{[15]}$

$$
\begin{gathered}
J(\hat{f})=\|Q \hat{f}\|^{2}+\alpha\left(\|g-H \hat{f}\|^{2}-\|n\|^{2}\right) \\
\hat{f}=\left(H^{T} H+\gamma Q^{T} Q\right)^{-1} H^{T} g
\end{gathered}
$$

$\gamma=\frac{1}{\alpha}$ is a constant that fulfil the constraint condition. $\alpha$ is the Lagrange coefficient, calculating partial differential of $\hat{f}$ in the first formula, and let the whole equation equal zero, obtaining $\hat{f}$.

\section{3) Image Denoising}

Noise often exists in the image, affecting the quality ${ }^{[3]}$ of the image and the effort of fuzzy image recovery. In order to contrast the image after recovery with the original image clearly, removing the noise is essential ${ }^{[12]}$.The change of pixel in the adjacent region is greater than the inside region in a image. That is, the change of the pixel in the middle region is less than the edge pixel. Therefore, the gradient is proportional to difference of the neighborhood pixel gray. Calculating the reciprocal of the gradient and using this reciprocal as the 
weighting factor, so the weight of the pixels within the region is greater than the external weight ${ }^{[7]}$.

TABLE III. THE DISTRIBUTION OF PIXEL

\begin{tabular}{|l|l|l|}
\hline$f(i-1, j-1)$ & $f(i-1, j)$ & $f(i-1, j+1)$ \\
\hline$f(i, j-1)$ & $f(i, j)$ & $f(i, j+1)$ \\
\hline$f(i+1, j-1)$ & $f(i+1, j)$ & $f(i+1, j+1)$ \\
\hline
\end{tabular}

$$
f(i, j, m, n)=\frac{1}{|f(i+m, j+n)-f(i, j)|}
$$

When $f(i+m, j+n)-f(i, j)=0, \quad f(i, j, m, n)=0$

Weight:

$$
t(i, j, m, n)=\frac{f(i, j, m, n)}{\sum_{m=-1}^{1} \sum_{n=1}^{1} f(i, j, m, n)} \times(1-r), m, n \neq 0
$$

Output inverse weighting filter:

$$
y=f(i, j) \times r+\sum_{m=-1}^{1} \sum_{n=1}^{1} t(i, j, m, n) \times f(i, j, m, n)
$$

$\mathrm{R}$ is the weight of the center pixel, $\mathrm{r}$ can be controlled

\section{E. Quality Testing of Image Restoration}

It is still impossible to match the original image completely after the restoration of the blurred image. So it is necessary to evaluate the reconstructed image according to the known original image. The main method is based on the error analysis method and the characteristic simulation of human visual system.

\section{1) Mean Error}

$$
M S E=\frac{1}{M N} \sum_{i} \sum_{j}\left|g_{(i, j)}-f_{(i, j)}\right|^{2}
$$

$M N$ represents the size of image, $f_{(i, j)}, g_{(i, j)}$ represent the gray value of the corresponding point of image $g$, $f$. The smaller value of $M S E$, the better of image quality.

\section{2) Gray Mean Grads}

To judge the quality of image restoration, it is possible by reflecting the details of the image, such as GMG (Gray Mean Grads), the larger value of $G M G$, the better and clearer of the image quality. But this method only uses the local area information can not consider the whole.

$$
\begin{gathered}
G M G=\frac{1}{M N} \sum_{i} \sum_{j} \sqrt{\frac{\Delta f_{x}^{2}+\Delta f_{y}^{2}}{2}} \\
=\frac{1}{M N} \sum_{i} \sum_{j} \sqrt{\frac{\left(f_{(x, y+1)}-f_{(x, y)}\right)^{2}+\left(f_{(x+1, y)}-f_{(x, y)}\right)^{2}}{2}}
\end{gathered}
$$

$f_{(x+1, y)}$ represent the grayscale value of images. The results of the quality evaluation of the original image and the restored image is $G M G_{1}$. If the smaller value of $G M G_{1}-G M G_{2}$ the better of image quality. On the contrary, the quality of image is poor.

\section{MODEL APPLICATION}

There are many blurred images in daily life, which bring people a lot of inconvenience. In recent years, the restoration of fuzzy images has become a hot spot. In the process of improving the image quality, the restoration of the images is an important step, and the image restoration model is widely used. Such as:

(1) Defective image restoration: because of the existence of noise and recording medium has defects, the image may have some information lost.

(2) Security field: criminal investigation traffic and safety monitoring, removing the noise in the target field of related images, enhancing the resolution of region of interest.

(3) Medical field: improving the resolution of medical images suppressing various noise of images X-ray CT.

(4) The field of astronomical imaging: For the imaging system on the ground, it will cause image degradation due to the impact of radiation and the atmosphere. The effort of atmospheric turbulence, defects of imaging detection system, various noise in the process of imaging.

(5) Other field: disposing of old photos recovery and quality improvement of early film, historical images and documents. The blurred images caused by defocusing and moving.TV telephony, remote diagnostics and other technologies rely on image quality.

\section{REFERENCES}

[1] Su Heng, Zhou Jie, Zhihao Zhang. Super-resolution image reconstruction [J]. Acta Automatica Sinica 2013, 08:1202-1213.

[2] Li Zhan, Qingfeng Zhang, Xiaohua Meng, Liang Peng, Yubao Liu. Super-resolution Reconstruction for Multi-resolution Image Sequence [J]. Acta Automatica Sinica 2012, 11:1804-1814.

[3] Xianyong Fang, Weiran Han, Zhou Jian, Li Li, Yanwen Guo, Deblurring of Motion Blurred Images Based on The Irradiance[J]. Journal of Image and Graphics, 2015, 03:395-407.

[4] Yuyue Zhou.Research of The Fractional Fourier Transform Applied in Optical Image Processing. [D] Harbin Institute of Technology, 2006.

[5] Xuefen Wang, Han Qin, Xueqin Jiang, Yan Chi,Jianglan Huang,Jiaxiang Cha.Research on Super-resolution Surveillance Image Reconstruction[J].Laser Journal,2014,03:5-8.

[6] Wang Lei. Image Registration Technology and Application [D] XiDian University, 2007

[7] Yiyan Wang. Research on Image De-noising Algorithm [D].Shaanxi Normal University, 2008.

[8] Liping Shen. The Research on Digital Image Processing Technology Based on Interpolation[D].Nanjing University of Aeronautics and Astronautics,2008.

[9] Jingxiao Shuai, Yongle Yan, Peng Yan, Jiangping Luo. An Improved Algorithm and Its Realization of Image Magnification Based on Bilinear Interpolation [J]. Nuclear Electronics \& Detection Technology, 2009, 01:55-58.

[10] Yaping Zhou. Studies on Image Registration Technique [D].XiDian University, 2008. 
[11] Wenze Shao. Image Modeling Based Regularized Multi-Frame Super-Resolution Reconstruction [D]. Nanjing University of Science and Technology, 2008.

[12] Xiaobo Zhang. Research of Image Denoising Algorithms Based on Wiener Filter [D].XiDian University, 2014.

[13] Xueqing Zhao. Research on Restoration of Degraded Image [D].Shanxi Normal University, 2013.

[14] Huang He. Research of The Image Denoising and Restoration Based on High-order Variation Method [D].Shenyang University, 2013.

[15] Zhang Rui. Image Restoration from Blurred Object of Linear Motion [D].Harbin Institute of Technology,2013. 